\title{
Observation of ECM Gap Phenomena through Transparent Electrode
}

\author{
Wenhao Zhang*, Tomoo Kitamura*, Masanori Kunieda*, Kohzoh Abe** \\ (Received on Jan. 1, 2014) \\ * Department of Precision Engineering, The University of Tokyo, Tokyo 113, Japan
** NIPPON STEEL \& SUMIKIN MATERIALS CO., LTD., Saitama 369, Japan
}

\begin{abstract}
During the process of electrochemical machining (ECM), bubbles and sludge are generated in the interelectrode gap, affecting the machining accuracy significantly. To solve this problem, an electrolyte flow is supplied into the gap to flush them away. Hence, this study aimed to observe the flow of electrolyte and bubbles in the gap using a transparent electrode. SiC single crystal semiconductor was used as the cathode material because it is electrically conductive and optically transparent. The gap was observed through the SiC cathode in the direction normal to the electrode surface using a high speed video camera. Distributions of the flow speed and diameter of bubbles were investigated to understand the influence of flushing flow on the machining process.
\end{abstract}

Key words: electrochemical machining (ECM), transparent electrode, SiC single crystal, bubble, electrolyte flushing flow

\section{INTRODUCTION}

In electrochemical machining (ECM), hydrogen gas bubbles and dissolution products (sludge of metal oxide) are generated in the machining gap. In the frontal gap, where the gap width is narrow, since accumulation of bubbles and sludge decreases the electrical conductivity of the electrolyte, the electrolytical dissolution is hindered. Hence, stray current flows in the side gap where the gap width is even wider, deteriorating the machining accuracy. Moreover, since Joule heating causes temperature rise in the gap, electrolyte may be even boiled [1][2]. To solve this problem, the electrolyte is supplied into the gap from a hole made in the tool electrode or workpiece. When the hole is not allowed to be made, the tool electrode is retracted cyclically to flush the gap. Use of pulsed current is another effective method to achieve high accuracy in ECM [3][4][5]. To understand the mechanism of improving the machining characteristics using the above technologies and further improve the machining characteristics of ECM, visualization of the fluid flow in the ECM gap is important. However, since the observation has been difficult, many simulation models have been proposed [2].

On the other hand, Kitamura et al. [6] observed the gap phenomena in electrical discharge machining (EDM) using transparent electrodes. Silicon carbide $(\mathrm{SiC})$ single crystal semiconductor was used as the electrode because it is electrically conductive and optically transparent. Hence, this study aims to observe the ECM gap phenomena through the transparent electrode using a high speed video camera and clarify the effect of the electrolyte flushing flow on the bubble behaviors.

\section{EXPERIMENTAL METHOD}

Fig. 1 shows the experimental setup used to observe the ECM gap phenomena in stationary electrolyte. The magnified image of the working gap was taken through a transparent electrode plate using a high-speed video camera (MEMRECAM HX-3, nac Image Technology Inc.). The machining cell was filled with $\mathrm{NaNO}_{3}$ aqueous solution of $5 \mathrm{wt} \%$. A rectangular transparent electrode plate and stainless steel (SUS 304) plate were used as the cathode and anode, respectively. The transparent electrode used in the present work was a single crystal silicon carbide (SiC) wafer of which the specifications are shown in Table 1. Its electrical conductivity was sufficiently high to be used as an electrode. When the SiC plate was used as an EDM electrode [6], the single crystal surface was thermally damaged due to discharge. Since the transparency was lost at discharge craters, observation of the EDM gap with a long time was difficult. ECM processes in contrast do not damage the $\mathrm{SiC}$ electrode.

Machining conditions used in the experiment are shown in Table 2. When the oscilloscope was triggered by the rise of the electrolytic current waveform measured by the current sensor, the oscilloscope output a trigger signal, which was sent to the high-speed video camera to freeze the memory of frames. Since the SiC plate was used as the cathode, hydrogen gas was generated on the $\mathrm{SiC}$ surface, while the stainless steel anode was dissolved. Table 3 shows the high speed video camera setting used in the experiments. 


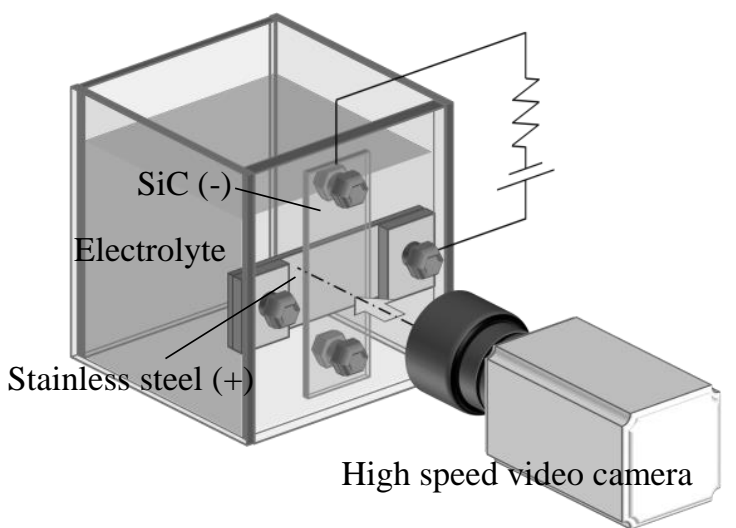

Fig. 1 Experimental setup to observe gap phenomena in stationary electrolyte

Table 1 Specifications of SiC single crystal wafers

\begin{tabular}{l|l}
\hline Manufacturer & NIPPON STEEL \& SUMIKIN \\
& MATERIALS CO., LTD. \\
Polytype & $4 \mathrm{H}$ \\
Surface orientation & $(0001)$ \\
Conduction type & N-type \\
Resistivity $[\Omega \mathrm{cm}]$ & $0.013 \sim 0.025$ \\
Thickness $[\mu \mathrm{m}]$ & 380 \\
\hline
\end{tabular}

Table 2 Machining conditions in stationary electrolyte

\begin{tabular}{l|lr}
\hline Polarity & $\begin{array}{l}\text { Cathode: SiC, } \\
\text { Anode: Stainless } \\
\text { (SUS304) }\end{array}$ \\
Gap width $[\mu \mathrm{m}]$ & 100,30 \\
Working area $\left[\mathrm{mm}^{2}\right]$ & $5 \times 5$ \\
Gap voltage $[\mathrm{V}]$ & 30 \\
Electrolyte & $\mathrm{NaNO}_{3}(\mathrm{aq})-5 \mathrm{wt} \%$ \\
\hline
\end{tabular}

Table 3 High speed video camera settings

\begin{tabular}{l|l}
\hline High speed video camera & $\begin{array}{l}\text { MEMRECAM HX-3, } \\
\text { nac Image Technology } \\
\text { Inc. }\end{array}$ \\
Resolution [pixel] & $960 \times 960$ \\
Frame rate [fps] & 10,000 \\
Exposure time [s] & $1 / 40,000$ \\
\hline
\end{tabular}

\section{GAP PHENOMENA IN STATIONARY ELECTROLYTE}

Fig. 2 shows the images of the working gap observed with different gap widths. The time indicates the passage after the voltage is applied to the gap. Fig. 3 shows the gap voltage and variation of electrolytic current with time. Before the voltage is applied, the anode surface can be seen through the clear electrolyte
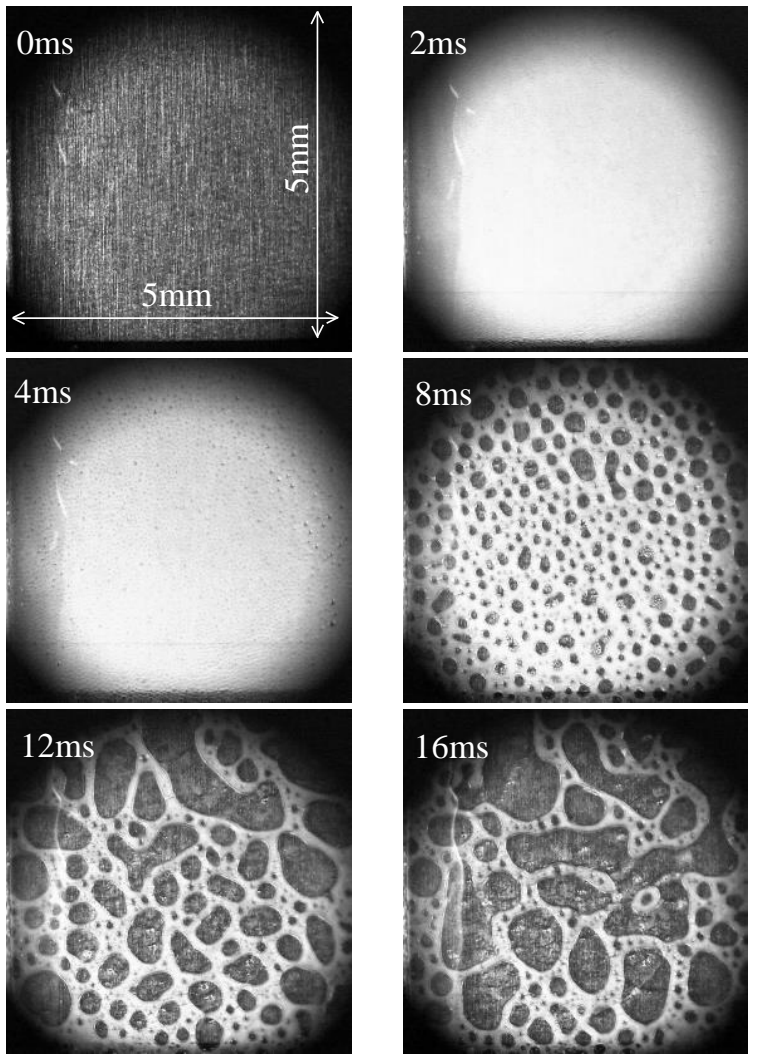

(a) Gap: $100 \mu \mathrm{m}$
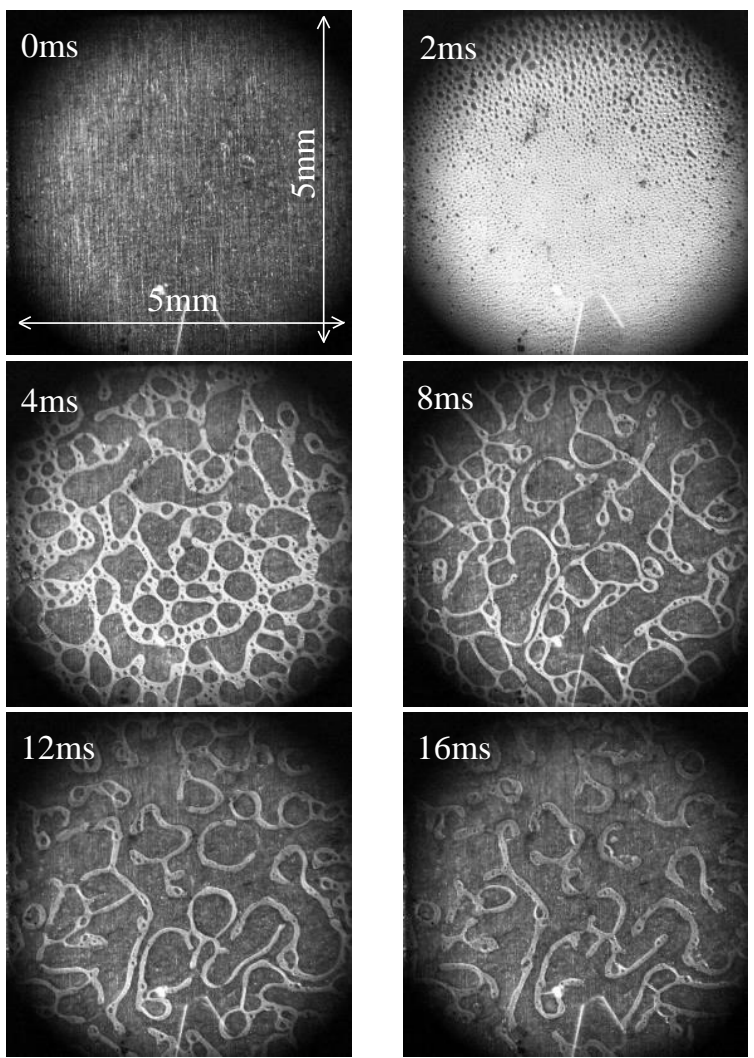

(b) Gap: $30 \mu \mathrm{m}$

Fig. 2 Generation of hydrogen bubbles in stationary electrolyte 
and transparent cathode. Immediately after the voltage is applied, the gap is filled with minute bubbles and the diameter of bubbles increases with time due to coalescence. Most of the gap was filled with bubbles within $16 \mathrm{~ms}$ and $8 \mathrm{~ms}$ when the gap width was 100 $\mu \mathrm{m}$ and $30 \mu \mathrm{m}$, respectively. Correspondingly, the electrolytic current decreases with time. The gap is filled with bubbles quicker with shorter gap width than with longer one because the volume of the gap space is smaller and the electrolytic current is larger with shorter gap width as shown in Fig. 3. These results demonstrate the importance of the flushing flow of electrolyte from the hollow space in the tool electrode as described in the next section. The results can also verify the fact that the use of pulsed current with duration of milliseconds order is useful to improve the machining accuracy of ECM [3][4][5]. In the experiments, there was no influence of gravity because the period of observation was significantly short.

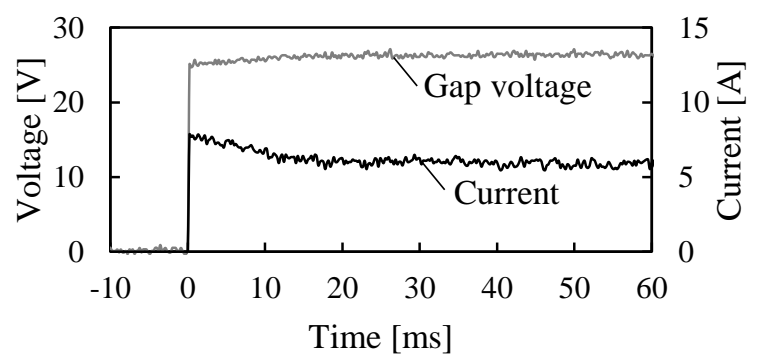

(a) Gap: $100 \mu \mathrm{m}$

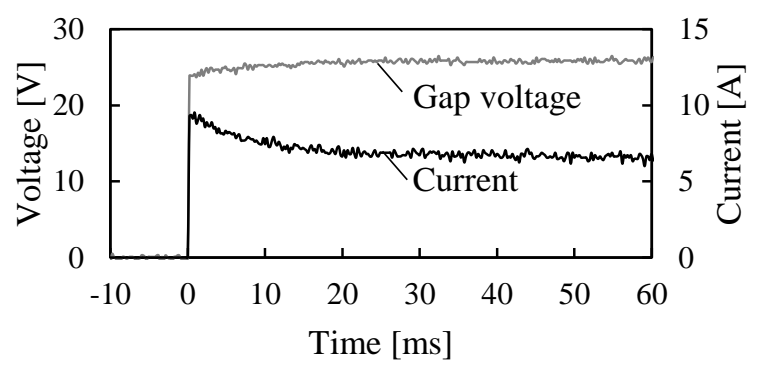

(b) Gap: $30 \mu \mathrm{m}$

Fig. 3 Waveforms of gap voltage and electrolytic current measured with different gap width

\section{EFFECT OF ELECTROLYTE FLUSHIING}

To see the effect of the flushing flow of electrolyte, the pair of cylindrical steel anode and disk SiC cathode shown in Fig. 4 were used in place of the rectangular electrodes in Fig. 1. The anode was a carbon steel disk of $14 \mathrm{~mm}$ in diameter. The electrolyte was jetted from the hollow space of the hole drilled at the center of the anode. The SiC cathode of $0.38 \mathrm{~mm}$ in thickness was adhered on the acrylic plate for reinforcement. The gap was observed through the acrylic plate and $\mathrm{SiC}$ cathode using the high speed camera. Machining conditions are shown in Table 4.

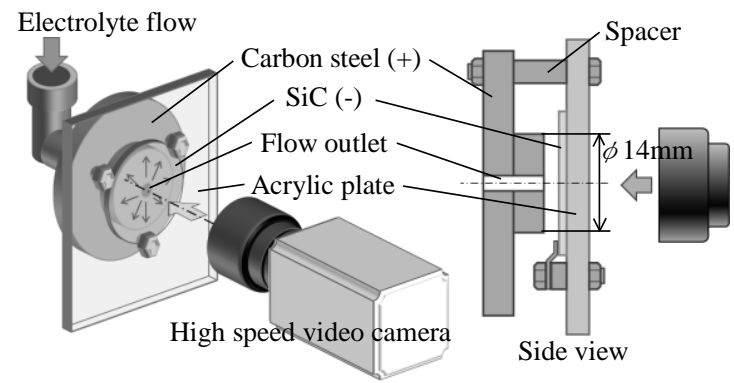

Fig. 4 Experimental setup to observe ECM gap with flushing flow

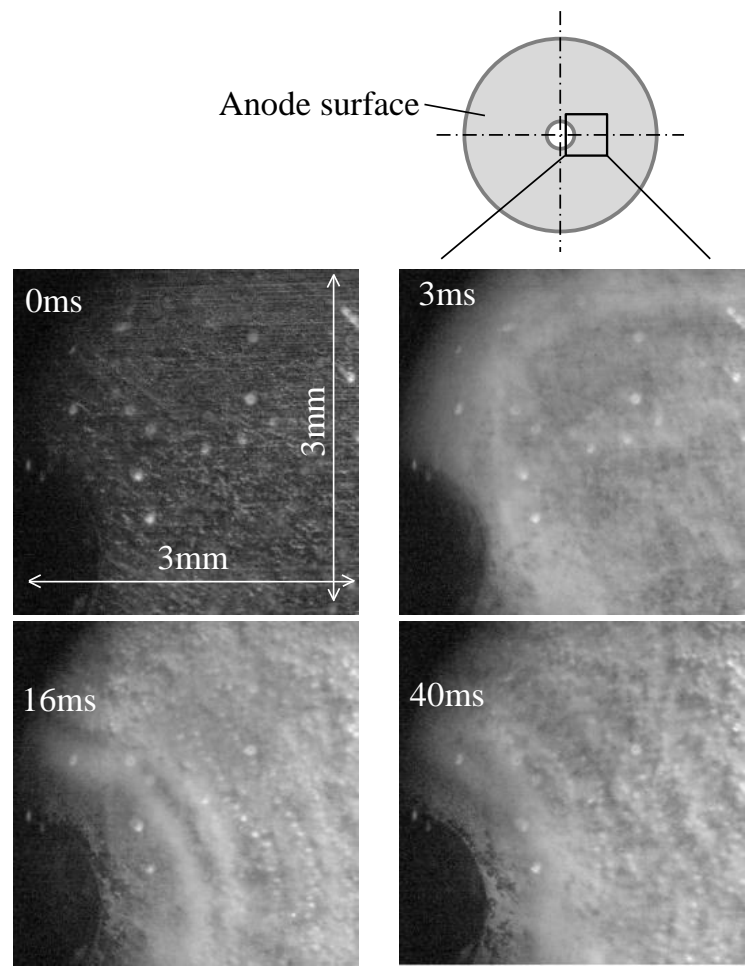

(a) With electrolyte flushing
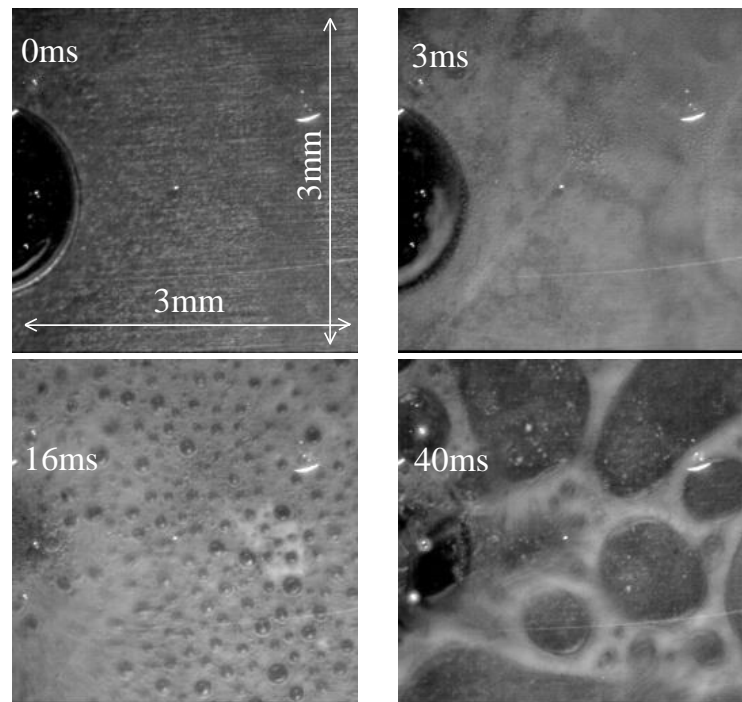

(b) Without electrolyte flushing

Fig. 5 Influence of electrolyte flushing on bubbles behavior 
Figs. 5 and 6 show the influences of electrolyte flushing on the bubbles behavior and electrolytic current, respectively. The size of bubbles existing in the gap is smaller with electrolyte flushing, because there is no sufficient time for bubbles to coalesce with each other before they get out of the working gap. The electrolytic current is kept constant with flushing, while it is reduced in the stationary electrolyte.

$\underline{\text { Table } 4 \text { Machining conditions with electrolyte flushing }}$

\begin{tabular}{l|l}
\hline Electrode polarity & $\begin{array}{l}\text { Cathode: } \mathrm{SiC}, \\
\text { Anode: Carbon steel } \\
(\mathrm{S} 45 \mathrm{C})\end{array}$ \\
Gap width $[\mu \mathrm{m}]$ & 165 \\
Gap voltage $[\mathrm{V}]$ & 30 \\
Working area $\left[\mathrm{mm}^{2}\right]$ & 150.8 \\
Current density $\left[\mathrm{A} / \mathrm{cm}^{2}\right]$ & 10 \\
Flow rate $[\mathrm{ml} / \mathrm{s}]$ & 3.35 \\
Electrolyte & $\mathrm{NaNO}_{3}(\mathrm{aq})-5 \mathrm{wt} \%$ \\
\hline
\end{tabular}

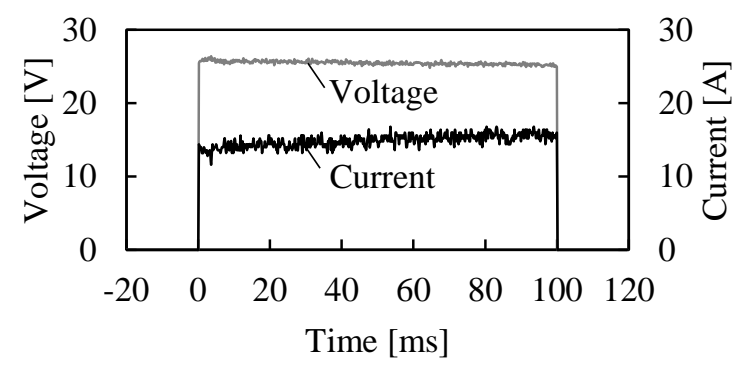

(a) With electrolyte flushing

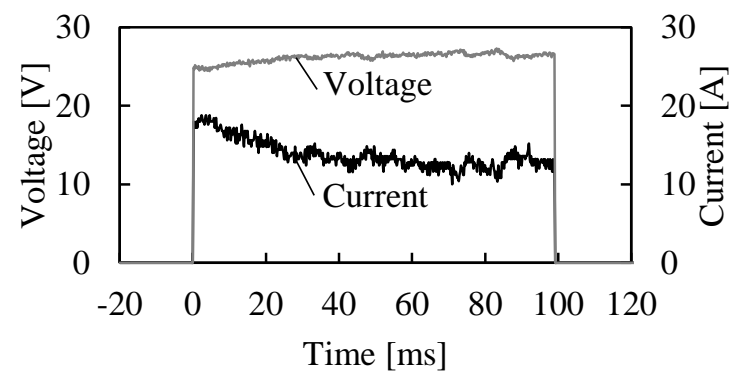

(b) Without electrolyte flushing

Fig. 6 Influence of electrolyte flushing on electrolytic current

\section{SPEED AND DIAMETER OF BUBBLES}

In the experiment with the flushing flow, the distributions of convection speed and diameter of the bubbles were investigated. To make the observation easy, the generation rate of bubbles was reduced by decreasing the gap voltage, and the convection speeds of bubbles were lowered by decreasing the electrolyte flow rate. The flow was observed in the downstream of the radial flow where the flow was laminar.
Machining conditions are shown in Table 5.

It was found from the experiment that most of the bubbles are moving in contact with the $\mathrm{SiC}$ cathode surface on which hydrogen gas is generated as shown in Fig. 7. Since the flow is laminar, bubbles cannot be detached from the surface. This was verified from the observed images which showed the shadows of bubbles on the anode surface. Since the illumination light was incident from the direction tilted from the direction normal to the $\mathrm{SiC}$ cathode surface, the distance of the center of each spherical bubble from the anode surface could be obtained from the position of the shadow.

Fig. 8 shows the change in the distribution of bubble speed with time. Immediately after the voltage is applied, all the bubbles which are visible are minute since they have not coalesced yet. Their speed is slow and uniform. With the lapse of time, dispersion of the speed increases. However, the minimum speed is the same. Fig. 9 shows the relationship between the bubble diameter and bubble speed observed. It is found that larger bubbles have higher speed. This relationship can be explained by the parabolic distribution of the electrolyte laminar flow in the gap shown in Fig. 10. Since bubbles are generated on the cathode surface, smaller bubbles exist in the boundary layer where the velocity is low. Thus, the mechanism of bubble coalescence can be explained as follows. Since larger bubbles have higher speeds, they can pass smaller bubbles, enabling coalescence.

Table 5 Machining conditions to investigate bubble speed

\begin{tabular}{l|l}
\hline Electrode polarity & $\begin{array}{l}\text { Cathode: } \mathrm{SiC}, \\
\text { Anode: Carbon steel } \\
(\mathrm{S} 45 \mathrm{C})\end{array}$ \\
Gap width $[\mu \mathrm{m}]$ & 120 \\
Gap voltage $[\mathrm{V}]$ & 10 \\
Working area $\left[\mathrm{mm}^{2}\right]$ & 150.8 \\
Current density $\left[\mathrm{A} / \mathrm{cm}^{2}\right]$ & 2.5 \\
Flow rate $\left[\mathrm{mm}^{3} / \mathrm{s}\right]$ & $1.45 \times 10^{3}$ \\
Electrolyte & $\mathrm{NaNO}_{3}(\mathrm{aq})-5 \mathrm{wt} \%$ \\
\hline
\end{tabular}

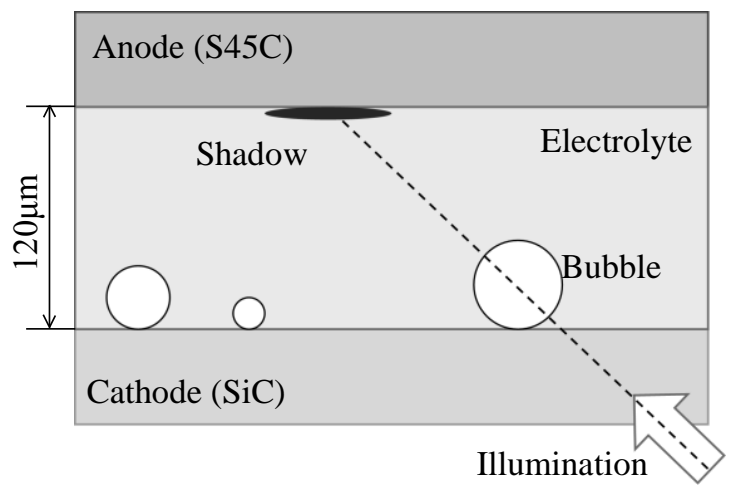

Fig. 7 Position of bubble center from electrode surfaces 


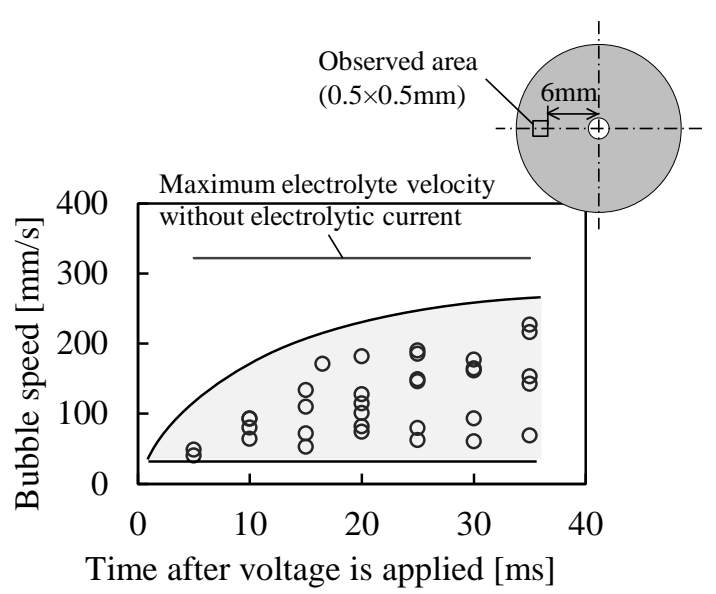

Fig. 8 Change in distribution of bubble speed

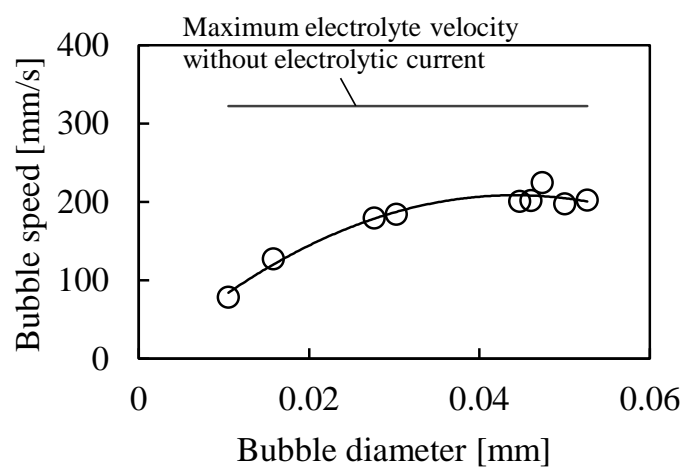

Fig. 9 Relationship between bubble diameter and bubble speed measured at $35 \mathrm{~ms}$

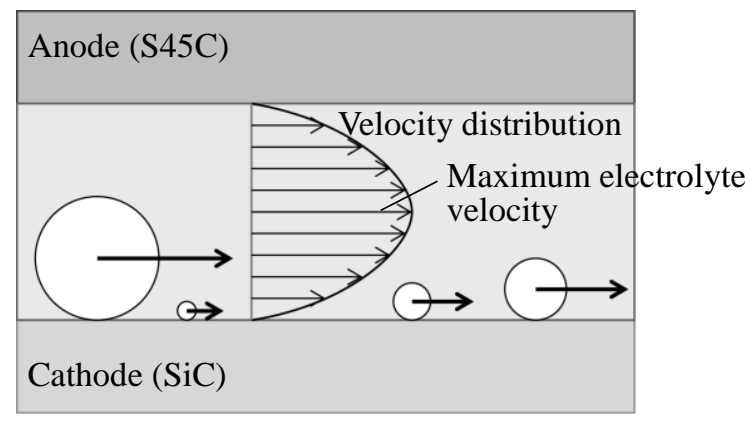

Fig. 10 Relationship between bubble speed and parabolic distribution of electrolyte flow field

\section{CONCLUSIONS}

The present work succeeded to visualize the ECM gap phenomena using $\mathrm{SiC}$ single crystal, which is electrically conductive and optically transparent. It is known that pulsed current with milliseconds in pulse duration is useful to improve the machining accuracy of ECM. However, the reason for this fact has not been explained quantitatively. Thus, using a high speed camera, hydrogen bubbles generated on the $\mathrm{SiC}$ cathode surface were observed. The following important gap flushing phenomena closely related to the machining stability were clarified.

(1) After the voltage was applied, most of the gap was filled with bubbles within $16 \mathrm{~ms}$ and $8 \mathrm{~ms}$ when the gap width was $100 \mu \mathrm{m}$ and $30 \mu \mathrm{m}$, respectively, in stationary electrolyte. This result indicates the advantage of the pulsed current with the pulse duration of milliseconds to improve the machining accuracy.

(2) The bubble diameter increases with time due to coalescence. The rate of increase is higher with smaller gap width.

(3) With the electrolyte flushing, diameters of bubbles are smaller than in stationary electrolyte because there is no sufficient time for bubbles to coalesce with each other, and the electrolytic current is kept constant.

(4) Since the bubbles are generated on the cathode surface in a laminar flow, larger bubbles have higher speed than smaller bubbles, resulting in bubble coalescence.

\section{ACKNOWLEDGEMENT}

The authors are grateful to Dr. T. Koyano of Kanazawa University, Mr. H. Sai of the University of Tokyo, and HODEN SEIMITSU KAKO KENKYUSHO CO., LTD., for their kind supports in conducting the experiments. This research was supported by the Grant-in-Aid from JSPS (25630020).

\section{REFERENCES}

[1] K. P. Rajurkar, D. Zhu, J. A. McGeough, J. Kozak, A. De Silva, New Developments in EletroChemical machining, Annals of the CIRP, 48, 2, 567-579 (1999).

[2] S. Hinduja, M. Kunieda, Modelling of ECM and EDM Processes, Annals of the CIRP, 62, 2, 775797 (2013).

[3] K. Chikamori, S. Ito, Electrochemical Machining in Stagnant Electrolyte, JSEME, 9, 18, 19-28 (1976) (in Japanese).

[4] K. P. Rajurkar, B. Wei, J. Kozak, Modelling and Monitoring Interelectrode Gap in Pulse Electrochemical Machining, Annals of the CIRP, 44, 1, 177-180 (1995).

[5] A. K. M. De Silva, H. S. J. Altena, J. A. McGeough, Precision ECM by Process Characteristic Modelling, Annals of the CIRP, 49, 1, 151-155 (2000).

[6] T. Kitamura, M. Kunieda, K. Abe, High-speed imaging of EDM gap phenomena using transparent electrodes, Proc. ISEM XVII, Procedia CIRP 6, 315-320 (2013). 